\title{
Bounded Holomorphic Functions Attaining their Norms in the Bidual
}

\author{
by \\ Daniel CARAndo and Martin Mazzitelli
}

\begin{abstract}
Under certain hypotheses on the Banach space $X$, we prove that the set of analytic functions in $\mathcal{A}_{u}(X)$ (the algebra of all holomorphic and uniformly continuous functions in the ball of $X$ ) whose Aron-Berner extensions attain their norms is dense in $\mathcal{A}_{u}(X)$. This Lindenstrauss type result also holds for functions with values in a dual space or in a Banach space with the so-called property $(\beta)$. We show that the Bishop-Phelps theorem does not hold for $\mathcal{A}_{u}\left(c_{0}, Z^{\prime \prime}\right)$ for a certain Banach space $Z$, while our Lindenstrauss theorem does. In order to obtain our results, we first handle their polynomial cases.
\end{abstract}

2010 Mathematics Subject Classification: Primary 47H60, 46G20; Secondary 46B28, $46 \mathrm{~B} 20$.

Keywords: integral formula, norm attaining holomorphic mappings, Lindenstrauss type theorems.

\section{$\S 1$. Introduction}

The study of the denseness of norm attaining mappings finds its origins in the Bishop-Phelps theorem [9], which asserts that the set of norm attaining bounded linear functionals on a Banach space is norm dense in the space of all bounded linear functionals. Since the appearance of this result in 1961, the study of norm attaining functions has attracted the attention of many authors. Given Banach spaces $X$ and $Y$, we say that a linear operator $T: X \rightarrow Y$ is norm attaining if there exists $x_{0}$ in the unit ball of $X$ such that $\left\|T\left(x_{0}\right)\right\|=\|T\|$. A question that arises naturally in this context is if it is possible to generalize the Bishop-Phelps theorem to bounded linear operators. The negative answer was given by Lindenstrauss [20].

Communicated by H. Okamoto. Received September 10, 2014. Revised February 6, 2015.

D. Carando: Departamento de Matemática - Pab I, Facultad de Ciencias Exactas y Naturales, Universidad de Buenos Aires, (1428) Buenos Aires, Argentina and IMAS-CONICET; e-mail: dcarando@dm.uba.ar

M. Mazzitelli: Departamento de Matemática - Pab I, Facultad de Ciencias Exactas y Naturales, Universidad de Buenos Aires, (1428) Buenos Aires, Argentina and IMAS-CONICET;

e-mail: mmazzite@dm.uba.ar 
On the other hand, he gave examples of Banach spaces $X$ for which the BishopPhelps theorem holds in $\mathcal{L}(X ; Y)$ for every Banach space $Y$. Such spaces are said to have property $A$. Similarly, a space $Y$ has property $B$ if the Bishop-Phelps theorem holds in $\mathcal{L}(X ; Y)$ for every $X$. A positive fundamental result given also in [20], the so-called Lindenstrauss theorem for linear operators, states that the set of bounded linear operators (between any two Banach spaces $X$ and $Y$ ) whose bitransposes are norm attaining, is dense in the space of all operators. This result was generalized by Acosta, García and Maestre [5] to multilinear operators, where the Bishop-Phelps theorem does not hold in general even in the scalar-valued case (we refer the reader to $[1,11,19]$ for counterexamples to the Bishop-Phelps theorem in the multilinear case). In this context, the role of the bitranspose is played by the canonical (Arens) extension to the bidual, obtained by weak-star density (see [6], $[15,1.9]$ and the definitions below).

In this paper, we study Lindenstrauss type theorems for polynomials and holomorphic functions. For 2-homogeneous scalar-valued polynomials, the Lindenstrauss theorem was proved in full generality by Aron, García and Maestre [8], where the Aron-Berner extension takes the place of the bitranspose. This result was later extended by Choi, Lee and Song [13] to vector-valued 2-homogeneous polynomials. In [11] a partial result was obtained for homogeneous polynomials of any degree. Specifically, if $X, Y$ are Banach spaces such that $X^{\prime}$ is separable and has the approximation property, then the set of $N$-homogeneous polynomials from $X$ to $Y^{\prime}$ whose Aron-Berner extensions attain their norms is dense in the set of all continuous $N$-homogeneous polynomials. It is worth noting that in the homogeneous case, there is no Bishop-Phelps theorem either; counterexamples can be found in $[11,19]$ for, respectively, scalar and vector-valued polynomials. Going further, we can ask about the validity of Bishop-Phelps and Lindenstrauss type theorems for non-homogeneous polynomials and holomorphic functions. In this direction, our main positive results are the following (see definitions and notation in Section 2).

Theorem A. Let $X$ be a Banach space whose dual is separable and has the approximation property. Suppose $W$ is a dual space or a Banach space with property $(\beta)$. Then the set of all polynomials of degree at most $k$ from $X$ to $W$ whose Aron-Berner extensions attain their norms is dense in the space of all continuous polynomials of degree at most $k$.

Theorem B. Let $X$ be a Banach space whose dual is separable and has the approximation property. Suppose $W$ is a dual space or a Banach space with property $(\beta)$. Then the set of all functions in $\mathcal{A}_{u}(X ; W)$ whose Aron-Berner extensions attain their norms is dense in $\mathcal{A}_{u}(X ; W)$. 
Theorems A and B are direct consequences of Theorem 3.2, Corollary 3.3 and Proposition 3.5. Indeed, Theorem 3.2 and Corollary 3.3 are just the part of Theorems A and B for mappings with values in dual spaces. In particular, they cover the scalar-valued case. Proposition 3.5 shows that if the Lindenstrauss theorem holds in the scalar-valued case, then it also holds with values in a Banach space with property $(\beta)$.

We also deal with stronger versions of Bishop-Phelps and Lindenstrauss theorems. Namely, we consider the density of mappings which attain their suprema in smaller balls, a problem studied, for example, by Acosta, Alaminos, García and Maestre [2]. We show in Section 3 that the strong versions of Theorems A and B also hold.

In Section 4 we show that, in general, there are no Bishop-Phelps theorems for scalar or vector-valued continuous polynomials (extending some known results) or for $\mathcal{A}_{u}(X ; Z)$. We remark that for the presented counterexamples, our Lindenstrauss theorem holds. We also address the strong variants of Bishop-Phelps and Lindenstrauss theorems, and show a counterexample to the strong Bishop-Phelps theorem in $\mathcal{A}_{u}(X)$.

\section{$\S 2$. Definitions and preliminary results}

Given a Banach space $X$, we denote by $X^{\prime}$ its dual space, while $B_{X}$ and $B_{X}^{\mathrm{o}}$ stand, respectively, for the closed and the open unit ball. By $\mathcal{L}\left(X_{1}, \ldots, X_{N} ; Y\right)$ we denote the space of all $N$-linear operators from $X_{1} \times \cdots \times X_{N}$ to $Y$. This space is endowed with the supremum norm

$$
\|\Phi\|=\sup \left\{\left\|\Phi\left(x_{1}, \ldots, x_{N}\right)\right\|: x_{i} \in B_{X_{i}}, 1 \leq i \leq N\right\} .
$$

We say that a multilinear operator $\Phi$ attains its norm if there exists an $N$-tuple $\left(a_{1}, \ldots, a_{N}\right) \in B_{X_{1}} \times \cdots \times B_{X_{N}}$ such that $\left\|\Phi\left(a_{1}, \ldots, a_{N}\right)\right\|=\|\Phi\|$.

Given $\Phi \in \mathcal{L}\left(X_{1}, \ldots, X_{N} ; Y\right)$, its Arens (or canonical) extension is the multilinear operator $\bar{\Phi}: X_{1}^{\prime \prime} \times \cdots \times X_{N}^{\prime \prime} \rightarrow Y^{\prime \prime}$ defined by

$$
\bar{\Phi}\left(x_{1}^{\prime \prime}, \ldots, x_{N}^{\prime \prime}\right)=w^{*}-\lim _{\alpha_{1}} \ldots \lim _{\alpha_{N}} \Phi\left(x_{1, \alpha_{1}}, \ldots, x_{N, \alpha_{N}}\right)
$$

where $\left(x_{i, \alpha_{i}}\right)_{\alpha_{i}} \subseteq X$ is a net $w^{*}$-convergent to $x_{i}^{\prime \prime} \in X_{i}^{\prime \prime}, i=1, \ldots, N$.

A continuous $N$-homogeneous polynomial is a function $P: X \rightarrow Y$ of the form $P(x)=\Phi(x, \ldots, x)$ for some continuous $N$-linear map $\Phi: X \times \cdots \times X \rightarrow Y$. We denote by $\mathcal{P}\left({ }^{N} X ; Y\right)$ the Banach space of all continuous $N$-homogeneous polynomials from $X$ to $Y$ endowed with the supremum norm

$$
\|P\|=\sup _{x \in B_{X}}\|P(x)\| .
$$


Naturally, we say that a polynomial $P$ is norm attaining if there exists $x_{0} \in B_{X}$ such that $\left\|P\left(x_{0}\right)\right\|=\|P\|$. The set of norm attaining $N$-homogeneous polynomials is denoted by $N A \mathcal{P}\left({ }^{N} X ; Y\right)$. We recall that the canonical extension of a polynomial $P \in \mathcal{P}\left({ }^{N} X ; Y\right)$ to the bidual, usually called the Aron-Berner extension [7], is the polynomial $\bar{P} \in \mathcal{P}\left({ }^{N} X^{\prime \prime} ; Y^{\prime \prime}\right)$ defined by $\bar{P}\left(x^{\prime \prime}\right)=\bar{\Phi}\left(x^{\prime \prime}, \ldots, x^{\prime \prime}\right)$, where $\Phi$ is the unique symmetric $N$-linear mapping associated to $P$.

Given $k \in \mathbb{N}$, let $\mathcal{P}_{k}(X ; Y)$ denote the Banach space of continuous polynomials from $X$ to $Y$ of degree less than or equal to $k$, endowed with the supremum norm. Each $P \in \mathcal{P}_{k}(X ; Y)$ can be written as $P=\sum_{j=0}^{k} P_{j}$, where each $P_{j}$ is a $j$ homogeneous polynomial. On the other hand, given a complex Banach space $X$, we denote by $\mathcal{A}_{u}(X ; Y)$ to the Banach space of holomorphic functions in the open unit ball $B_{X}^{\mathrm{o}}$ which are uniformly continuous in the closed unit ball $B_{X}$, endowed with the supremum norm. It is well-known that each $f \in \mathcal{A}_{u}(X ; Y)$ is a uniform limit of polynomials. When $Y=\mathbb{K}$ is the scalar field, we simply write $\mathcal{P}_{k}(X)$ or $\mathcal{A}_{u}(X)$. As expected, a function $f$ in $\mathcal{P}_{k}(X ; Y)$ or $\mathcal{A}_{u}(X ; Y)$ is said to be norm attaining if there exists $x_{0} \in B_{X}$ such that $\left\|f\left(x_{0}\right)\right\|=\|f\|$, and the subsets of norm attaining functions are denoted by $N A \mathcal{P}_{k}(X ; Y)$ and $N A \mathcal{A}_{u}(X ; Y)$. The Aron-Berner extension of a polynomial $P=\sum_{j=0}^{k} P_{j} \in \mathcal{P}_{k}(X ; Y)$ is given by $\bar{P}=\sum_{j=0}^{k} \overline{P_{j}}$. In the case of a function $f \in \mathcal{A}_{u}(X ; Y)$, given its Taylor series expansion at $0, f=\sum_{j=0}^{\infty} P_{j}$, the Aron-Berner extension of $f$ is defined as $\bar{f}=$ $\sum_{j=0}^{\infty} \overline{P_{j}}$, which is a holomorphic function in the open unit ball $B_{X^{\prime \prime}}^{\mathrm{o}}[14]$. Note that if $\left(P_{n}\right)_{n \in \mathbb{N}}$ is a sequence of polynomials converging uniformly to $f$, then $\left(\overline{P_{n}}\right)_{n \in \mathbb{N}}$ is uniformly Cauchy in the ball $B_{X^{\prime \prime}}^{\circ}$, and then converges uniformly to $\bar{f}$. This means that $\bar{f}$ extends to a uniformly continuous function in the closed unit ball of $X^{\prime \prime}$. Davie and Gamelin [14] showed that $\|\bar{f}\|=\|f\|$ in the scalar-valued case. The same holds for a vector-valued $f \in \mathcal{A}_{u}(X ; Y)$, since $\bar{f}\left(x^{\prime \prime}\right)\left(y^{\prime}\right)=\overline{y^{\prime} \circ f}\left(x^{\prime \prime}\right)$ for all $x^{\prime \prime} \in X^{\prime \prime}$ and $y^{\prime} \in Y^{\prime}$.

Throughout the article, in the polynomial results the scalar field can be either $\mathbb{R}$ or $\mathbb{C}$, while we consider only complex Banach spaces in the holomorphic setting.

\section{Duality for non-homogeneous polynomials}

Polynomials in $\mathcal{P}\left({ }^{j} X\right)$ can be thought of as continuous linear functionals on the symmetric projective tensor product as follows. Given a symmetric tensor $u_{j}$ in $\otimes^{j, s} X$ (the $j$-fold symmetric tensor product of $X$ ), the symmetric projective norm $\pi_{s}$ of $u_{j}$ is defined by

$$
\pi_{s}\left(u_{j}\right)=\inf \left\{\sum_{i=1}^{m}\left|\lambda_{i}\right|\left\|x_{i}\right\|^{j}: u_{j}=\sum_{i=1}^{m} \lambda_{i} x_{i}^{j},\left(\lambda_{i}\right)_{i=1}^{m} \subset \mathbb{K},\left(x_{i}\right)_{i=1}^{m} \subset X\right\} .
$$

We denote by $\tilde{\otimes}_{\pi_{s}}^{j, s} X$ the completion of $\otimes^{j, s} X$ with respect to $\pi_{s}$. Then $\mathcal{P}\left({ }^{j} X\right)=$ 
$\left(\tilde{\otimes}_{\pi_{s}}^{j, s} X\right)^{\prime}$ isometrically, where the identification is given by the duality

$$
L_{p_{j}}\left(u_{j}\right):=\left\langle u_{j}, p_{j}\right\rangle=\sum_{i=1}^{\infty} \lambda_{i} p_{j}\left(x_{i}\right),
$$

for $p_{j} \in \mathcal{P}\left({ }^{j} X\right)$ and $u_{j} \in \tilde{\otimes}_{\pi_{s}}^{j, s} X, u_{j}=\sum_{i=1}^{\infty} \lambda_{i} x_{i}^{j}$.

Consider the space

$$
G_{k}=\bigoplus_{j=0}^{k}\left(\tilde{\otimes}_{\pi_{s}}^{j, s} X\right)
$$

where we set $\tilde{\otimes}_{\pi_{s}}^{0, s} X=\mathbb{K}$. An element $u \in G_{k}$ is of the form $u=\sum_{j=0}^{k} u_{j}$ with $u_{j} \in \tilde{\otimes}_{\pi_{s}}^{j, s} X$. We endow this space with the norm

$$
\|u\|_{G_{k}}=\sup _{q \in B_{\mathcal{P}_{k}(X)}}\left|\sum_{j=0}^{k}\left\langle u_{j}, q_{j}\right\rangle\right|,
$$

where $q_{j}$ is the $j$-homogeneous part of $q$. It is easy to check that $\left(G_{k},\|\cdot\|_{G_{k}}\right)$ is a Banach space.

With the previous notation, given $p \in \mathcal{P}_{k}(X)$ we have $\left\|p_{j}\right\| \leq\|p\|$ for every $0 \leq j \leq k$ as a consequence of the Cauchy inequalities. Therefore, for $u_{j} \in \tilde{\otimes}_{\pi_{s}}^{j, s} X$ we get

$$
\left\|0+\cdots+u_{j}+\cdots+0\right\|_{G_{k}}=\sup _{q \in B_{\mathcal{P}_{k}(X)}}\left|\left\langle u_{j}, q_{j}\right\rangle\right| \leq \sup _{q \in B_{\mathcal{P}_{k}(X)}}\left\|u_{j}\right\|_{\pi_{s}}\left\|q_{j}\right\| \leq\left\|u_{j}\right\|_{\pi_{s}} .
$$

We have shown the following.

Remark 2.1. The space $\mathcal{P}\left({ }^{j} X\right)$ is 1-complemented in $\mathcal{P}_{k}(X)$. Also, $\tilde{\otimes}_{\pi_{s}}^{j, s} X$ is 1-complemented in $G_{k}$.

The following lemma shows that $G_{k}$ linearizes polynomials of degree at most $k$.

Lemma 2.2. Let $X$ be a Banach space and $k \in \mathbb{N}$. The mapping

$$
\mathcal{P}_{k}(X) \rightarrow\left(G_{k},\|\cdot\|_{G_{k}}\right)^{\prime}, \quad p \mapsto L_{p}
$$

where $L_{p}(u)=\langle u, p\rangle=\sum_{j=0}^{k}\left\langle u_{j}, p_{j}\right\rangle$, is an isometric isomorphism.

Proof. Let us see that it is an isometry. By the previous remark,

$$
\left|L_{p}(u)\right|=\|p\|\left|\sum_{j=0}^{k}\left\langle u_{j}, p_{j} /\|p\|\right\rangle\right| \leq\|p\|\|u\|_{G_{k}}
$$


which implies that $L_{p} \in G_{k}^{\prime}$ with $\left\|L_{p}\right\| \leq\|p\|$. Now, given $\varepsilon>0$ take $x_{0} \in B_{X}$ with $\left|p\left(x_{0}\right)\right|>\|p\|-\varepsilon$, and consider

$$
u_{0}=\sum_{j=0}^{k} \underbrace{x_{0} \otimes \cdots \otimes x_{0}}_{j} .
$$

Then $u_{0} \in B_{G_{k}}$ and $\left|L_{p}\left(u_{0}\right)\right|=\left|p\left(x_{0}\right)\right|>\|p\|-\varepsilon$, which gives the reverse inequality.

Now we prove that the mapping (2) is surjective. For $L \in G_{k}^{\prime}$, let $L_{j}$ denote its restriction to $\tilde{\otimes}_{\pi_{s}}^{j, s} X$, that is,

$$
L_{j}:=L_{\tilde{\otimes}_{\pi_{s}}^{j, s} X}: \tilde{\otimes}_{\pi_{s}}^{j, s} X \rightarrow \mathbb{K}, \quad L_{j}\left(u_{j}\right)=L\left(0+\cdots+u_{j}+\cdots+0\right) .
$$

It is clear that $L_{j}$ is linear and, by Remark 2.1, $\left|L\left(u_{j}\right)\right| \leq\|L\|\left\|u_{j}\right\|_{\pi_{s}}$ for each $u_{j} \in \tilde{\otimes}_{\pi_{s}}^{j, s} X$. Then $L_{j} \in\left(\tilde{\otimes}_{\pi_{s}}^{j, s} X\right)^{\prime}$ and we can find $p_{j} \in \mathcal{P}\left({ }^{j} X\right)$ such that $L_{j}=L_{p_{j}}$. Now, if we take $p=p_{0}+\cdots+p_{k} \in \mathcal{P}_{k}(X)$ it is easy to check that $L=L_{p}$.

For polynomials with values in a dual space $Y^{\prime}$ we have the isometric isomorphism

$$
\mathcal{P}\left({ }^{j} X ; Y^{\prime}\right)=\left(\left(\tilde{\otimes}_{\pi_{s}}^{j, s} X\right) \tilde{\otimes}_{\pi} Y\right)^{\prime} .
$$

Here the duality is given by

$$
L_{P_{j}}\left(u_{j}\right):=\left\langle u_{j}, P_{j}\right\rangle=\sum_{l=1}^{\infty} \sum_{i=1}^{\infty} \lambda_{l, i} P_{j}\left(x_{l, i}\right)\left(y_{l}\right)
$$

for any $P_{j} \in \mathcal{P}\left({ }^{j} X ; Y^{\prime}\right)$ and $u_{j}=\sum_{l=1}^{\infty} v_{l} \otimes y_{l}$, where $\left(y_{l}\right)_{l} \subset Y$ and $\left(v_{l}\right)_{l} \subset \tilde{\otimes}_{\pi_{s}}^{j, s} X$, with $v_{l}=\sum_{i=1}^{\infty} \lambda_{l, i} x_{l, i}^{j}$ for all $l$.

We define

$$
G_{k}=\bigoplus_{j=0}^{k}\left(\left(\tilde{\otimes}_{\pi_{s}}^{j, s} X\right) \tilde{\otimes}_{\pi} Y\right),
$$

where the elements are of the form $u=\sum_{j=0}^{k} u_{j}$ with $u_{j} \in\left(\tilde{\otimes}_{\pi_{s}}^{j, s} X\right) \tilde{\otimes}_{\pi} Y$. The norm of such an element is given by

$$
\|u\|_{G_{k}}=\sup _{Q \in B_{\mathcal{P}_{k}\left(X ; Y^{\prime}\right)}}\left|\sum_{j=0}^{k}\left\langle u_{j}, Q_{j}\right\rangle\right| .
$$

Now the duality

$$
\mathcal{P}_{k}\left(X ; Y^{\prime}\right) \stackrel{1}{=}\left(G_{k},\|\cdot\|_{G_{k}}\right)^{\prime}
$$

is defined exactly as in Lemma 2.2, that is, $P \mapsto L_{P}$ where $L_{P}(u)=\langle u, P\rangle$. 
Note that if we consider the space

$$
\bigoplus_{j=0}^{\infty}\left(\left(\tilde{\otimes}_{\pi_{s}}^{j, s} X\right) \tilde{\otimes}_{\pi} Y\right)
$$

of all elements $u \in G_{k}$ for any $k \in \mathbb{N} \cup\{0\}$, then for each $f=\sum_{j=0}^{\infty} P_{j} \in$ $H^{\infty}\left(B_{X}^{\mathrm{o}} ; Y^{\prime}\right)$ and $u=\sum_{j=0}^{k} u_{j}$ we have the duality $\langle u, f\rangle=\sum_{j=0}^{k}\left\langle u_{j}, P_{j}\right\rangle$. We endow this space with the norm

$$
\|u\|=\sup _{g \in B_{H^{\infty}\left(B_{X}^{\mathrm{o}} ; Y^{\prime}\right)}}|\langle u, g\rangle|,
$$

and we denote its completion by $G_{\infty}$. An easy calculation shows that the map $f \mapsto L_{f}$, where $L_{f}(u)=\langle u, f\rangle$, defines an isometric isomorphism giving the duality

$$
H^{\infty}\left(B_{X}^{\mathrm{o}} ; Y^{\prime}\right) \stackrel{1}{=} G_{\infty}^{\prime}
$$

We have obtained, in a somewhat different way, the space $G_{\infty}$ constructed by Mujica [22]. Actually, what we have is a description of this space in terms of tensor products.

\section{§3. An integral formula and Lindenstrauss type theorems}

In this section we will prove the main results of the article, summarized in Theorems $\mathrm{A}$ and $\mathrm{B}$ in the Introduction. The following result extends [11, Theorem 2.2] to the non-homogeneous setting.

Lemma 3.1. Let $X, Y$ be Banach spaces and suppose that $X^{\prime}$ is separable and has the approximation property. Then for each $u \in G_{k}$ there exists a regular Borel measure $\mu_{u}$ on $\left(B_{X^{\prime \prime}}, w^{*}\right) \times\left(B_{Y^{\prime \prime}}, w^{*}\right)$ such that $\left\|\mu_{u}\right\| \leq\|u\|_{G_{k}}$ and

$$
\langle u, P\rangle=\int_{B_{X^{\prime \prime}} \times B_{Y^{\prime \prime}}} \bar{P}\left(x^{\prime \prime}\right)\left(y^{\prime \prime}\right) d \mu_{u}\left(x^{\prime \prime}, y^{\prime \prime}\right)
$$

for all $P \in \mathcal{P}_{k}\left(X ; Y^{\prime}\right)$.

Proof. We first prove the formula for the set $\mathcal{P}_{f, k}\left(X ; Y^{\prime}\right)$ of finite type polynomials of degree less than or equal to $k$, that is, for the polynomials of the form $P=$ $P_{0}+\cdots+P_{k}$ where the $j$-homogeneous polynomial $P_{j}$ is a linear combination of polynomials of the form $x^{\prime}(\cdot)^{j} \cdot y$. Given $u \in G_{k}$ we define

$$
\Lambda_{u}: \mathcal{P}_{f, k}\left(X ; Y^{\prime}\right) \rightarrow \mathbb{K}, \quad \Lambda_{u}(P)=\langle u, P\rangle .
$$


It is easily verified that $\left\|\Lambda_{u}\right\| \leq\|u\|_{G_{k}}$. The finite type polynomials can be seen as an isometric subspace of $C\left(B_{X^{\prime \prime}} \times B_{Y^{\prime \prime}}\right)$, where the balls are endowed with their weak-star topologies, by identifying a polynomial $P \in \mathcal{P}_{f, k}\left(X ; Y^{\prime}\right)$ with the function $\left(x^{\prime \prime}, y^{\prime \prime}\right) \mapsto \bar{P}\left(x^{\prime \prime}\right)\left(y^{\prime \prime}\right)$. Then we extend $\Lambda_{u}$ by the Hahn-Banach theorem to a continuous linear functional on $C\left(B_{X^{\prime \prime}} \times B_{Y^{\prime \prime}}\right)$ preserving the norm. Now, by the Riesz representation theorem, there is a regular Borel measure $\mu_{u}$ on $\left(B_{X^{\prime \prime}}, w^{*}\right) \times\left(B_{Y^{\prime \prime}}, w^{*}\right)$ such that $\left\|\mu_{u}\right\| \leq\|u\|_{G_{k}}$ and

$$
\Lambda_{u}(f)=\int_{B_{X^{\prime \prime}} \times B_{Y^{\prime \prime}}} f\left(x^{\prime \prime}, y^{\prime \prime}\right) d \mu_{u}\left(x^{\prime \prime}, y^{\prime \prime}\right)
$$

for $f \in C\left(B_{X^{\prime \prime}} \times B_{Y^{\prime \prime}}\right)$, where we still use $\Lambda_{u}$ for its extension to $C\left(B_{X^{\prime \prime}} \times B_{Y^{\prime \prime}}\right)$. In particular, taking $f=P \in \mathcal{P}_{f, k}\left(X ; Y^{\prime}\right)$ we obtain the integral formula for finite type polynomials.

Now, take $P=P_{0}+\cdots+P_{k} \in \mathcal{P}_{k}\left(X ; Y^{\prime}\right)$. By [11, Lemma 2.1], for each $P_{j}$, $0 \leq j \leq k$, there exists a norm bounded multi-indexed sequence of finite type polynomials $\left(P_{j, n_{1}, \ldots, n_{j}}\right)_{\left(n_{1}, \ldots, n_{j}\right) \in \mathbb{N}^{j}}$ satisfying

$$
\overline{P_{j}}\left(x^{\prime \prime}\right)\left(y^{\prime \prime}\right)=\lim _{n_{1} \rightarrow \infty} \ldots \lim _{n_{j} \rightarrow \infty} \overline{P_{j, n_{1}, \ldots, n_{j}}}\left(x^{\prime \prime}\right)\left(y^{\prime \prime}\right) .
$$

For fixed $0 \leq j \leq k$ we define $P_{j, n_{1}, \ldots, n_{k}}:=P_{j, n_{1}, \ldots, n_{j}}$ for all $n_{j+1}, \ldots, n_{k} \in \mathbb{N}$. Then the multi-indexed sequences $\left(P_{j, n_{1}, \ldots, n_{k}}\right)_{\left(n_{1}, \ldots, n_{k}\right) \in \mathbb{N}^{k}}$ are indexed on the same index set and satisfy

$$
\overline{P_{j}}\left(x^{\prime \prime}\right)\left(y^{\prime \prime}\right)=\lim _{n_{1} \rightarrow \infty} \ldots \lim _{n_{k} \rightarrow \infty} \overline{P_{j, n_{1}, \ldots, n_{k}}}\left(x^{\prime \prime}\right)\left(y^{\prime \prime}\right) .
$$

Now, consider $P_{n_{1}, \ldots, n_{k}}=\sum_{j=0}^{k} P_{j, n_{1}, \ldots, n_{k}} \in \mathcal{P}_{f, k}\left(X ; Y^{\prime}\right)$. Since the integral formula holds for finite type polynomials, we have

$$
\left\langle u, P_{n_{1}, \ldots, n_{k}}\right\rangle=\int_{B_{X^{\prime \prime}} \times B_{Y^{\prime \prime}}} \overline{P_{n_{1}, \ldots, n_{k}}}\left(x^{\prime \prime}\right)\left(y^{\prime \prime}\right) d \mu_{u}\left(x^{\prime \prime}, y^{\prime \prime}\right),
$$

for all $\left(n_{1}, \ldots, n_{k}\right) \in \mathbb{N}^{k}$. As the sequence $\left(P_{n_{1}, \ldots, n_{k}}\right)_{\left(n_{1}, \ldots, n_{k}\right) \in \mathbb{N}^{k}}$ is norm bounded, we may apply the bounded convergence theorem $k$ times to obtain

$$
\begin{aligned}
\lim _{n_{1} \rightarrow \infty} \ldots \lim _{n_{k} \rightarrow \infty} & \left\langle u, P_{n_{1}, \ldots, n_{k}}\right\rangle \\
& =\lim _{n_{1} \rightarrow \infty} \ldots \lim _{n_{k} \rightarrow \infty} \int_{B_{X^{\prime \prime}} \times B_{Y^{\prime \prime}}} \overline{P_{n_{1}, \ldots, n_{k}}}\left(x^{\prime \prime}\right)\left(y^{\prime \prime}\right) d \mu_{u}\left(x^{\prime \prime}, y^{\prime \prime}\right) \\
& =\int_{B_{X^{\prime \prime}} \times B_{Y^{\prime \prime}}} \bar{P}\left(x^{\prime \prime}\right)\left(y^{\prime \prime}\right) d \mu_{u}\left(x^{\prime \prime}, y^{\prime \prime}\right) .
\end{aligned}
$$

It remains to show that $\langle u, P\rangle=\lim _{n_{1} \rightarrow \infty} \ldots \lim _{n_{k} \rightarrow \infty}\left\langle u, P_{n_{1}, \ldots, n_{k}}\right\rangle$. Note that, for each $0 \leq j \leq k$, both $\left\langle\cdot, P_{j}\right\rangle$ and $\lim _{n_{1} \rightarrow \infty} \ldots \lim _{n_{k} \rightarrow \infty}\left\langle\cdot, P_{j, n_{1}, \ldots, n_{k}}\right\rangle$ 
are continuous linear functions on $\left(\tilde{\otimes}_{\pi_{s}}^{j, s} X\right) \tilde{\otimes}_{\pi} Y$ which coincide on elementary tensors. Since $P=\sum_{j=0}^{k} P_{j}$ and $P_{n_{1}, \ldots, n_{k}}=\sum_{j=0}^{k} P_{j, n_{1}, \ldots, n_{k}}$ the claim follows and the proof is complete.

Now we are ready to state our Lindenstrauss type theorem for non-homogeneous polynomials. We sketch the proof of the statement which is similar to that of [11, Theorem 2.3].

Theorem 3.2. Let $X, Y$ be Banach spaces. Suppose that $X^{\prime}$ is separable and has the approximation property. Then the set of all polynomials in $\mathcal{P}_{k}\left(X ; Y^{\prime}\right)$ whose Aron-Berner extensions attain their norms is dense in $\mathcal{P}_{k}\left(X ; Y^{\prime}\right)$.

Proof. Given $Q \in \mathcal{P}_{k}\left(X ; Y^{\prime}\right)$ consider its associated linear function $L_{Q} \in G_{k}^{\prime}$, defined as in Lemma 2.2. The Bishop-Phelps theorem asserts that, for $\varepsilon>0$, there exists a norm attaining functional $L=L_{P} \in G_{k}^{\prime}$ such that $\left\|L_{Q}-L_{P}\right\|<\varepsilon$ for some polynomial $P$ in $\mathcal{P}_{k}\left(X ; Y^{\prime}\right)$. Since $\left\|L_{Q}-L_{P}\right\|=\|Q-P\|$, it remains to prove that $\bar{P}$ is norm attaining.

Take $u \in G_{k}$ such that $\|u\|_{G_{k}}=1$ and $\left|L_{P}(u)\right|=\left\|L_{P}\right\|=\|P\|$, and take the regular Borel measure $\mu_{u}$ on $B_{X^{\prime \prime}} \times B_{Y^{\prime \prime}}$ given by Lemma 3.1. Then

$$
\|P\|=\left|L_{P}(u)\right| \leq \int_{B_{X^{\prime \prime}} \times B_{Y^{\prime \prime}}}\left|\bar{P}\left(x^{\prime \prime}\right)\left(y^{\prime \prime}\right)\right| d\left|\mu_{u}\right|\left(x^{\prime \prime}, y^{\prime \prime}\right) \leq\|\bar{P}\|\left\|\mu_{u}\right\| \leq\|P\| .
$$

Consequently, $\left|\bar{P}\left(x^{\prime \prime}\right)\left(y^{\prime \prime}\right)\right|=\|P\|$ almost everywhere (for $\left.\mu_{u}\right)$. Hence $\bar{P}$ attains its norm.

Since functions in $\mathcal{A}_{u}\left(X ; Y^{\prime}\right)$ are uniform limits of polynomials, and each polynomial, by the previous theorem, is close to a polynomial whose Aron-Berner extension is norm attaining, we obtain the following Lindenstrauss theorem for the space $\mathcal{A}_{u}\left(X ; Y^{\prime}\right)$.

Corollary 3.3. Let $X, Y$ be Banach spaces. Suppose that $X^{\prime}$ is separable and has the approximation property. Then the set of all functions in $\mathcal{A}_{u}\left(X ; Y^{\prime}\right)$ whose Aron-Berner extensions attain their norms is dense in $\mathcal{A}_{u}\left(X ; Y^{\prime}\right)$. Moreover, given $g \in \mathcal{A}_{u}\left(X ; Y^{\prime}\right)$ and $\varepsilon>0$ there exists a polynomial $P$ such that $\bar{P}$ is norm attaining and $\|g-P\|<\varepsilon$.

In order to obtain more examples of spaces on which the Lindenstrauss theorem holds, we bring up the so-called property $(\beta)$, which was introduced by Lindenstrauss [20], who also showed that it implies property $B$ (see comments in the Introduction). In other words, if a space $Y$ has property $(\beta)$ then the Bishop-Phelps theorem holds in $\mathcal{L}(X ; Y)$ for every Banach space $X$. In the real finite-dimensional 
case, the spaces with property $(\beta)$ are precisely those whose unit ball is a polyhedron. In the infinite-dimensional case, examples of these spaces are $c_{0}, \ell_{\infty}$ and $C(K)$ with $K$ having a dense set of isolated points. We recall the definition.

Definition 3.4. A Banach space $Y$ has property $(\beta)$ if there exists a subset $\left\{\left(y_{\alpha}, g_{\alpha}\right): \alpha \in \Lambda\right\} \subset Y \times Y^{\prime}$ satisfying:

(i) $\left\|y_{\alpha}\right\|=\left\|g_{\alpha}\right\|=g_{\alpha}\left(y_{\alpha}\right)=1$.

(ii) There exists $\lambda, 0 \leq \lambda<1$, such that $\left|g_{\alpha}\left(y_{\beta}\right)\right| \leq \lambda$ for $\alpha \neq \beta$.

(iii) For all $y \in Y,\|y\|=\sup _{\alpha \in \Lambda}\left|g_{\alpha}(y)\right|$.

Following the ideas of [20, Proposition 3], Choi and Kim [12, Theorem 2.1] proved that if the Bishop-Phelps theorem holds in $\mathcal{P}\left({ }^{N} X\right)$, then it holds in $\mathcal{P}\left({ }^{N} X ; Y\right)$ for every space $Y$ with property $(\beta)$. Mimicking their ideas we can prove an analogous statement for the Lindenstrauss theorem; we give a proof for completeness. Since there are spaces with property $(\beta)$ which are not dual spaces, this gives new examples of spaces satisfying a Lindenstrauss theorem.

Proposition 3.5. Suppose that $Y$ has property $(\beta)$. If the Lindenstrauss theorem holds for $\mathcal{P}\left({ }^{N} X\right)$ (respectively $\mathcal{P}_{k}(X), \mathcal{A}_{u}(X)$ ) then it also holds for $\mathcal{P}\left({ }^{N} X ; Y\right)$ (respectively $\mathcal{P}_{k}(X ; Y), \mathcal{A}_{u}(X ; Y)$ ).

Proof. We prove the $N$-homogeneous case since the others are completely analogous. Consider $Q \in \mathcal{P}\left({ }^{N} X ; Y\right)$ and $\varepsilon>0$. We may suppose $\|Q\|=1$ without loss of generality. Note that since $Y$ has property $(\beta)$, we easily get $1=\|Q\|=$ $\sup _{\alpha}\left\|g_{\alpha} \circ Q\right\|$ and we can take $\alpha_{0}$ such that $\left\|g_{\alpha_{0}} \circ Q\right\| \geq 1-\varepsilon(1-\lambda) / 4$. By hypothesis there exists $p \in \mathcal{P}\left({ }^{N} X\right)$ with $\|p\|=\left\|g_{\alpha_{0}} \circ Q\right\|$, such that $\left\|g_{\alpha_{0}} \circ Q-p\right\|<$ $\varepsilon(1-\lambda) / 2$ and $\bar{p}$ attains the norm, say, at $x_{0}^{\prime \prime} \in B_{X^{\prime \prime}}$. Define $P \in \mathcal{P}\left({ }^{N} X ; Y\right)$ by $P(x)=Q(x)+\left((1+\varepsilon) p(x)-g_{\alpha_{0}} \circ Q(x)\right) y_{\alpha_{0}}$ and note that

$$
\|Q-P\| \leq \varepsilon\|p\|+\left\|g_{\alpha_{0}} \circ Q-p\right\| \leq \varepsilon+\varepsilon(1-\lambda) \leq 2 \varepsilon .
$$

It remains to see that $\bar{P}$ is norm attaining. For this purpose, we need first to prove that $\|P\|=\left\|g_{\alpha_{0}} \circ P\right\|$. Note that $\|P\|=\sup _{\alpha}\left\|g_{\alpha} \circ P\right\|$ and that given any $\alpha$ we have

$$
\begin{aligned}
\left\|g_{\alpha} \circ P\right\| & \leq\left\|g_{\alpha} \circ Q\right\|+\left|g_{\alpha}\left(y_{\alpha_{0}}\right)\right|\left(\varepsilon\|p\|+\left\|p-g_{\alpha_{0}} \circ Q\right\|\right) \\
& \leq 1+\lambda(\varepsilon+\varepsilon(1-\lambda) / 2) \leq 1+\varepsilon(1+\lambda) / 2 .
\end{aligned}
$$

On the other hand, since $g_{\alpha_{0}} \circ P=(1+\varepsilon) p$ and $\|p\|=\left\|g_{\alpha_{0}} \circ Q\right\| \geq 1-\varepsilon(1-\lambda) / 4$, we have

$$
\left\|g_{\alpha_{0}} \circ P\right\| \geq(1+\varepsilon)(1-\varepsilon(1-\lambda) / 4) \geq 1+\varepsilon(1+\lambda) / 2,
$$


which, together with the previous inequality, gives $\|P\|=\left\|g_{\alpha_{0}} \circ P\right\|$. Noting that $\overline{g_{\alpha_{0}} \circ P}\left(x^{\prime \prime}\right)=\bar{P}\left(x^{\prime \prime}\right)\left(g_{\alpha_{0}}\right)$ and recalling that $\bar{p}$ attains the norm at $x_{0}^{\prime \prime}$, we obtain

$$
\begin{aligned}
\|P\| & =\left\|g_{\alpha_{0}} \circ P\right\|=(1+\varepsilon)\|p\|=(1+\varepsilon)\left|\bar{p}\left(x_{0}^{\prime \prime}\right)\right| \\
& =\left|\bar{P}\left(x_{0}^{\prime \prime}\right)\left(g_{\alpha_{0}}\right)\right| \leq\left\|\bar{P}\left(x_{0}^{\prime \prime}\right)\right\| \leq\|P\| .
\end{aligned}
$$

This proves that $\bar{P}$ is norm attaining, and the result follows.

\section{A strong version of the Lindenstrauss theorem}

Up to our knowledge, it is still unknown if the Bishop-Phelps theorem holds for $\mathcal{A}_{u}(X)$. In [2], a different version of the Bishop-Phelps theorem is shown to fail for $\mathcal{A}_{u}(X)$. Namely, given $0<s \leq 1$ and $f \in \mathcal{A}_{u}(X)$ we define

$$
\|f\|_{s}=\sup \{|f(x)|:\|x\| \leq s\},
$$

which is clearly a norm on $\mathcal{A}_{u}(X)$; note that for $s=1$ we get the usual supremum norm denoted by $\|\cdot\|$. Then, we can ask about the denseness of functions that attain the $\|\cdot\|_{s}$-norm. Note that given $0<s \leq s_{0} \leq 1$, if the $\|\cdot\|_{s}$-norm attaining functions are $\|\cdot\|_{s_{0}}$-dense (that is, dense in the $\|\cdot\|_{s_{0}}$-norm) in $\mathcal{A}_{u}(X)$, then the BishopPhelps theorem holds. Indeed, given $g \in \mathcal{A}_{u}(X)$ and $\varepsilon>0$ take a polynomial $q$ such that $\|g-q\|<\varepsilon / 2$ and consider $q_{1 / s}$ defined by $q_{1 / s}(\cdot)=q\left(\frac{1}{s} \cdot\right)$. By the assumption, we have a $\|\cdot\|_{s}$-norm attaining function $f \in \mathcal{A}_{u}(X)$ such that $\left\|q_{1 / s}-f\right\|_{s_{0}}<\varepsilon / 2$. If we define $f_{s} \in \mathcal{A}_{u}(X)$ by $f_{s}(\cdot)=f(s \cdot)$, then $f_{s}$ is $\|\cdot\|$-norm attaining and $\left\|f_{s}\right\|=\|f\|_{s}$. On the other hand, $\left\|q-f_{s}\right\|=\left\|q_{1 / s}-f\right\|_{s} \leq\left\|q_{1 / s}-f\right\|_{s_{0}}<\varepsilon / 2$, and consequently $\left\|g-f_{s}\right\|<\varepsilon$. The same holds in the vector-valued case.

We will refer to these type of results (i.e., the denseness of functions that attain the $\|\cdot\|_{s}$-norm) as strong versions of the Bishop-Phelps theorem. When these stronger versions come on the scene, we will specify carefully whether we consider the $\|\cdot\|$-norm or some $\|\cdot\|_{s}$-norm; otherwise, the usual supremum norm is taken. The following result will be improved in Section 4, where also the definition of the preduals of Lorentz sequence spaces will be given.

Theorem 3.6 ([2, Corollary 4.5]). Let $X=d_{*}(w, 1)$ with $w \in \ell_{2} \backslash \ell_{1}$. Given $0<$ $s<1 / e$, the set of elements of $\mathcal{A}_{u}(X)$ that attain the $\|\cdot\|_{s}-$ norm is not $\|\cdot\|$-dense in $\mathcal{A}_{u}(X)$.

Taking this result into account, it is natural to ask if a Lindenstrauss theorem holds for the $\|\cdot\|_{s}$-norm in $\mathcal{A}_{u}\left(X ; Y^{\prime}\right)$. Our goal now is to give a partial positive answer to this problem. We briefly sketch the arguments, since they are slight modifications of those followed in the first part of this section. First, we state the following more general version of the well-known Bishop-Phelps theorem (see [10] or the final comment added in [9]). 
( $\star$ Let $X$ be a real Banach space, $C \subseteq X$ a bounded closed convex set and

$$
C^{*}=\left\{\varphi \in X^{\prime}: \varphi\left(x_{0}\right)=\sup _{x \in C} \varphi(x) \text { for some } x_{0} \in C\right\} .
$$

Then $C^{*}$ is dense in $X^{\prime}$. If $X$ is a real or complex Banach space and $C \subseteq X$ is bounded, closed, convex and balanced, then the set $C^{*}=\left\{\varphi \in X^{\prime}:\left|\varphi\left(x_{0}\right)\right|=\right.$ $\sup _{x \in C}|\varphi(x)|$ for some $\left.x_{0} \in C\right\}$ is dense in $X^{\prime}$.

Given Banach spaces $X, Y$ and $0<s \leq 1$, recall the predual $G_{k}$ of $\mathcal{P}_{k}\left(X ; Y^{\prime}\right)$ defined in Section 2 and consider the subset

$$
C_{s}=\left\{u \in G_{k}: \sup _{\|Q\|_{s} \leq 1}|\langle u, Q\rangle| \leq 1\right\},
$$

which turns to be a bounded, closed, balanced and convex set. It is easily verified that $\sup _{u \in C_{s}}\left|L_{P}(u)\right|=\|P\|_{s}$ for any $P \in \mathcal{P}_{k}\left(X ; Y^{\prime}\right)$. Also, if we take $P_{s}(\cdot)=P(s \cdot)$, it can be checked that $(\bar{P})_{s}=\overline{P_{s}}$ and $\|\bar{P}\|_{s}=\|P\|_{s}$. For elements in $C_{s}$, we have the following generalization of the integral formula presented in Lemma 3.1. The proof is analogous, just taking the $\|\cdot\|_{s}$-norm in the subspace of finite type polynomials instead of the usual supremum norm.

Lemma 3.7. Let $0<s \leq 1$ and let $X, Y$ be Banach spaces such that $X^{\prime}$ is separable and has the approximation property. Then for each $u \in C_{s}$ there exists a regular Borel measure $\mu_{u}$ on $\left(s B_{X^{\prime \prime}}, w^{*}\right) \times\left(B_{Y^{\prime \prime}}, w^{*}\right)$ such that $\left\|\mu_{u}\right\| \leq 1$ and

$$
\langle u, P\rangle=\int_{s B_{X^{\prime \prime}} \times B_{Y^{\prime \prime}}} \bar{P}\left(x^{\prime \prime}\right)\left(y^{\prime \prime}\right) d \mu_{u}\left(x^{\prime \prime}, y^{\prime \prime}\right)
$$

for all $P \in \mathcal{P}_{k}\left(X ; Y^{\prime}\right)$.

We now state our strong version of the Lindenstrauss theorem, generalizing the Lindenstrauss type results obtained in Corollary 3.3 and Proposition 3.5. The proof is analogous to the corresponding results for the supremum norm, making use of the more general versions of the Bishop-Phelps theorem $(\star)$ and the integral formula (6) of Lemma 3.7.

Theorem 3.8. Let $0<s \leq 1$ and suppose that $X^{\prime}$ is separable and has the approximation property. Then, for $W$ being either a dual space or a Banach space with property $(\beta)$, the set of polynomials from $X$ to $W$ whose Aron-Berner extensions attain their $\|\cdot\|_{s}$-norms is $\|\cdot\|$-dense in $\mathcal{A}_{u}(X ; W)$.

If $0<s \leq s_{0} \leq 1, W$ is a dual space or has property $(\beta)$, and $X^{\prime}$ is separable and has the approximation property, the previous theorem trivially implies the $\|\cdot\|_{s_{0}}$-denseness in $\mathcal{A}_{u}(X ; W)$ of the polynomials whose Aron-Berner extensions attain their $\|\cdot\|_{s}$-norms. In particular, the set of polynomials whose Aron-Berner 
extensions attain their $\|\cdot\|_{s}$-norms is $\|\cdot\|_{s}$-dense in $\mathcal{A}_{u}(X ; W)$. This seemingly stronger version of Corollary 3.3 and Proposition 3.5 is actually equivalent. Indeed, take $g \in \mathcal{A}_{u}(X ; W)$ and $\varepsilon>0$. Consider $g_{s} \in \mathcal{A}_{u}(X ; W)$ defined by $g_{s}(\cdot)=g(s \cdot)$. By the assumption there exists a polynomial $P$ such that $\bar{P}$ is $\|\cdot\|$-norm attaining and $\left\|g_{s}-P\right\|<\varepsilon$. Take $P_{1 / s}(\cdot)=P\left(\frac{1}{s} \cdot\right)$ and note that $\left\|P_{1 / s}\right\|_{s}=\|P\|$ and $\overline{P_{1 / s}}$ is $\|\cdot\|_{s}$-norm attaining. On the other hand, it is easy to see that $\left\|g-P_{1 / s}\right\|_{s}=$ $\left\|g_{s}-P\right\|<\varepsilon$.

Note that for $g \in H^{\infty}\left(B_{X}^{\mathrm{o}} ; W\right)$ and $0<s_{0}<1$, the function $g_{s_{0}}(\cdot)=g\left(s_{0} \cdot\right)$ belongs to $\mathcal{A}_{u}(X ; W)$. As a consequence of the previous theorem, given $0<s \leq$ $s_{0}<1$, if $X^{\prime}$ is separable and has the approximation property and $W$ is a dual space or has property $(\beta)$, then the set of polynomials whose Aron-Berner extensions attain their $\|\cdot\|_{s}$-norms is $\|\cdot\|_{s_{0}}$-dense in $H^{\infty}\left(B_{X}^{\mathrm{o}} ; W\right)$. We do not know whether the same is true for $s_{0}=1$.

\section{$\S 4$. Counterexamples to Bishop-Phelps theorems}

The preduals of Lorentz sequence spaces appear related to the study of denseness of norm attaining functions as a useful tool in finding counterexamples to the BishopPhelps type theorems. Gowers [16] was the first to use such a predual to prove that the spaces $\ell_{p}(1<p<\infty)$ do not have the property $B$ of Lindenstrauss. Later, the same space was used in [1] to show the failure of the Bishop-Phelps theorem for bilinear forms and 2-homogeneous scalar-valued polynomials. In [19], the authors characterize those preduals of Lorentz sequence spaces in which the Bishop-Phelps theorem holds for multilinear forms and $N$-homogeneous scalarvalued polynomials.

We now recall some definitions and properties (for further details on Lorentz sequence spaces, see [21, Chapter 4.e]). An admissible sequence will mean a decreasing sequence $w=\left(w_{i}\right)_{i \in \mathbb{N}}$ of non-negative real numbers with $w_{1}=1, \lim w_{i}=0$ and $\sum_{i} w_{i}=\infty$. The real or complex Lorentz sequence space $d(w, 1)$ associated to an admissible sequence $w=\left(w_{i}\right)_{i \in \mathbb{N}}$ is the vector space of all bounded sequences $x=(x(i))_{i}$ such that

$$
\|x\|_{w, 1}:=\sum_{i=1}^{\infty} x^{*}(i) w_{i}<\infty,
$$

where $x^{*}=\left(x^{*}(i)\right)_{i}$ is the decreasing rearrangement of $(x(i))_{i}$. This is a nonreflexive Banach space when is endowed with the norm $\|\cdot\|_{w, 1}$. It is known that the predual of $d(w, 1)$, denoted by $d_{*}(w, 1)$, is the space of all sequences $x$ such that

$$
\lim _{n \rightarrow \infty} \frac{\sum_{i=1}^{n} x^{*}(i)}{W(n)}=0
$$


where $W(n)=\sum_{i=1}^{n} w_{i}$. In this space the norm is defined by

$$
\|x\|_{W}:=\sup _{n} \frac{\sum_{i=1}^{n} x^{*}(i)}{W(n)}<\infty .
$$

Note that the condition $w_{1}=1$ is equivalent to the assumption that $\left\|e_{i}\right\|_{W}=1$ for all $i$ in $\mathbb{N}$, where $e_{i}$ stands for the canonical $i$-th vector of $d_{*}(w, 1)$.

There are two fundamental properties of the spaces $d_{*}(w, 1)$, which make them important in the study of these topics. The first one is related to the geometry of the unit ball, more precisely the lack of extreme points. The second is about the inclusion of these spaces in $\ell_{r}$ whenever the admissible sequence $w$ belongs to $\ell_{r}$. We state these properties below; their proofs can be found, for instance, in [19, Lemma 2.2 and Proposition 2.4].

- Given $x \in B_{d_{*}(w, 1)}$, there exist $n_{0} \in \mathbb{N}$ and $\delta>0$ such that $\left\|x+\lambda e_{n}\right\|_{W} \leq 1$ for all $|\lambda| \leq \delta$ and $n \geq n_{0}$.

- If $w \in \ell_{r}, 1<r<\infty$, then the formal inclusion $d_{*}(w, 1) \hookrightarrow \ell_{r}$ is bounded.

It is important to mention that preduals of Lorentz sequence spaces have a shrinking basis and hence satisfy the hypotheses of the Lindenstrauss type theorems proved in Section 3. From now on, $w$ will denote an admissible sequence.

\section{Counterexamples in the polynomial case}

Let us summarize some known results about bounds on the derivatives of polynomials (see, for instance, [17], [18]).

Lemma 4.1. Let $X$ and $Y$ be Banach spaces over the scalar field $\mathbb{K}=\mathbb{R}$ or $\mathbb{C}$. For fixed natural numbers $1 \leq j \leq k$, there exists a constant $C_{k, j}>0$ (depending only on $j$ and $k$ ) such that

$$
\left\|\frac{D^{j} P(x)}{j !}\right\| \leq C_{k, j}\|P\|
$$

for every $P \in \mathcal{P}_{k}(X ; Y)$ and $x \in B_{X}$

The following results extend [19, Lemma 3.1 and Theorem 3.2] to the nonhomogeneous case.

Lemma 4.2. Let $X$ be a complex Banach sequence space and $W$ be a strictly convex Banach space. Suppose that a polynomial $P: X \rightarrow W$ attains the norm at an element $x_{0} \in B_{X}$ satisfying the following condition:

$$
\exists n_{0} \in \mathbb{N} \text { and } \delta>0 \text { such that } \quad\left\|x_{0}+\lambda e_{n}\right\| \leq 1, \quad \forall|\lambda| \leq \delta \text { and } n \geq n_{0} .
$$

Then $D^{j} P\left(x_{0}\right)\left(e_{n}\right)=0$ for all $j \geq 1$ and $n \geq n_{0}$. 
Proof. Fix $n \geq n_{0}$. Since $P$ attains its norm at $x_{0}$, the modulus of the one-variable holomorphic function

$$
\{|\lambda|<\delta\} \rightarrow W, \quad \lambda \mapsto P\left(x_{0}+\lambda e_{n}\right),
$$

attains a local maximum at the origin. By the maximum modulus principle, this function must be constant. Let us see that this implies that $D^{j} P\left(x_{0}\right)\left(e_{n}\right)=0$ for all $j \geq 1$. Consider the series expansion of $P$ at $x_{0}$,

$$
P(x)=\sum_{j=0}^{\infty} \frac{D^{j} P\left(x_{0}\right)}{j !}\left(x-x_{0}\right) .
$$

Evaluating at $x=x_{0}+\lambda e_{n}$ and recalling that $\lambda \mapsto P\left(x_{0}+\lambda e_{n}\right)$ is a constant function we obtain

$$
P\left(x_{0}\right)=P\left(x_{0}+\lambda e_{n}\right)=P\left(x_{0}\right)+\sum_{j=1}^{\infty} \frac{D^{j} P\left(x_{0}\right)}{j !}\left(e_{n}\right) \lambda^{j}
$$

for all $|\lambda|<\delta$. Then $0=\sum_{j=1}^{\infty} \frac{D^{j} P\left(x_{0}\right)}{j !}\left(e_{n}\right) \lambda^{j}$ for all $|\lambda|<\delta$, and consequently $D^{j} P\left(x_{0}\right)\left(e_{n}\right)=0$ for all $j \geq 1$.

Proposition 4.3. Given an admissible sequence $w$ and $N \geq 2$, the following statements are equivalent:

(i) $N A \mathcal{P}\left({ }^{N} d_{*}(w, 1)\right)$ is dense in $\mathcal{P}\left({ }^{N} d_{*}(w, 1)\right)$.

(ii) If $k \geq N$, then every $N$-homogeneous polynomial in $\mathcal{P}\left({ }^{N} d_{*}(w, 1)\right)$ can be approximated by norm attaining polynomials in $\mathcal{P}_{k}\left(d_{*}(w, 1)\right)$.

(iii) $w \notin \ell_{N}$

Proof. The implication (i) $\Rightarrow$ (ii) is trivial, while (iii) $\Rightarrow$ (i) follows from $[19$, Theorem $3.2]$. Let us show that (ii) $\Rightarrow$ (iii). We suppose that $w \in \ell_{N}$ and give different proofs for $d_{*}(w, 1)$ complex or real Banach space.

The complex case. Take $q \in \mathcal{P}\left({ }^{N} d_{*}(w, 1)\right)$ defined by $q(x)=\sum_{i=1}^{\infty} x(i)^{N}$ (here we use $\left.d_{*}(w, 1) \hookrightarrow \ell_{N}\right)$. If $p \in \mathcal{P}_{k}\left(d_{*}(w, 1)\right)$ attains its norm at some $x_{0} \in B_{d_{*}(w, 1)}$, then Lemma 4.2 ensures that $D^{N} p\left(x_{0}\right)\left(e_{n}\right)=0$ for $n$ sufficiently large. Since $D^{N} q\left(x_{0}\right) / N$ ! $=q$, by Lemma 4.1 we obtain, for large $n$,

$$
1=\left|\frac{D^{N} q\left(x_{0}\right)}{N !}\left(e_{n}\right)-\frac{D^{N} p\left(x_{0}\right)}{N !}\left(e_{n}\right)\right| \leq\left\|\frac{D^{N} q\left(x_{0}\right)}{N !}-\frac{D^{N} p\left(x_{0}\right)}{N !}\right\| \leq C_{k, N}\|q-p\| .
$$

Therefore, $q$ cannot be approximated by norm attaining polynomials.

The real case. Let $M \leq N$ be the smallest natural number such that $w \in \ell_{M}$, consider $q \in \mathcal{P}\left({ }^{N} d_{*}(w, 1)\right)$ defined by $q(x)=x(1)^{N-M} \sum_{i=1}^{\infty}(-1)^{i} x(i)^{M}$ and suppose 
that $q$ can be approximated by norm attaining polynomials in $\mathcal{P}_{k}\left(d_{*}(w, 1)\right)$. Fix $\varepsilon>0$ and, applying Lemma 4.1, take $p \in N A \mathcal{P}_{k}\left(d_{*}(w, 1)\right)$ such that

$$
\left\|\frac{D^{M} q(x)}{M !}-\frac{D^{M} p(x)}{M !}\right\|<\varepsilon
$$

for any $x \in B_{d_{*}(w, 1)}$. Now, let $x_{0} \in B_{d_{*}(w, 1)}$ be such that $\|p\|=\left|p\left(x_{0}\right)\right|$ and take $n_{0} \in \mathbb{N}$ and $\delta>0$ so that (7) is satisfied. Assume for the moment that $p\left(x_{0}\right)>0$.

Then

$$
p\left(x_{0}+\lambda e_{n}\right)=p\left(x_{0}\right)+\sum_{j=1}^{k} \frac{D^{j} p\left(x_{0}\right)}{j !}\left(e_{n}\right) \lambda^{j} \leq p\left(x_{0}\right)
$$

for $|\lambda|<\delta$ and $n \geq n_{0}$. In view of [19, Theorem 3.2], for any $j<M$ the $j$ homogeneous polynomial $D^{j} p\left(x_{0}\right) / j$ ! is weakly sequentially continuous and consequently $\lim _{n \rightarrow \infty}\left(D^{j} p\left(x_{0}\right) / j !\right)\left(e_{n}\right)=0$. Then, taking limits in (9) and dividing by $\lambda^{M}$ we obtain

$$
\limsup _{n \rightarrow \infty} \sum_{j=M}^{k} \frac{D^{j} p\left(x_{0}\right)}{j !}\left(e_{n}\right) \lambda^{j-M} \leq 0
$$

for $0 \leq \lambda<\delta$. As a consequence,

$$
\limsup _{n \rightarrow \infty} \frac{D^{M} p\left(x_{0}\right)}{M !}\left(e_{n}\right) \leq 0 .
$$

If $p\left(x_{0}\right)<0$, reasoning with $-p$ we get

$$
\liminf _{n \rightarrow \infty} \frac{D^{M} p\left(x_{0}\right)}{M !}\left(e_{n}\right) \geq 0 .
$$

On the other hand, an easy calculation shows that

$$
\limsup _{n \rightarrow \infty} \frac{D^{M} q\left(x_{0}\right)}{M !}\left(e_{n}\right)=\left|x_{0}(1)\right|^{N-M}=-\liminf _{n \rightarrow \infty} \frac{D^{M} q\left(x_{0}\right)}{M !}\left(e_{n}\right) .
$$

Now, by (8), if $p\left(x_{0}\right)>0$ we have

$$
\left|x_{0}(1)\right|^{N-M}=\limsup _{n \rightarrow \infty} \frac{D^{M} q\left(x_{0}\right)}{M !}\left(e_{n}\right) \leq \limsup _{n \rightarrow \infty} \frac{D^{M} p\left(x_{0}\right)}{M !}\left(e_{n}\right)+\varepsilon \leq \varepsilon,
$$

while if $p\left(x_{0}\right)<0$ then

$$
-\left|x_{0}(1)\right|^{N-M}=\liminf _{n \rightarrow \infty} \frac{D^{M} q\left(x_{0}\right)}{M !}\left(e_{n}\right) \geq \liminf _{n \rightarrow \infty} \frac{D^{M} p\left(x_{0}\right)}{M !}\left(e_{n}\right)-\varepsilon \geq-\varepsilon .
$$


Therefore,

$$
\begin{aligned}
\|q\| & \leq\|p\|+\varepsilon=\left|p\left(x_{0}\right)\right|+\varepsilon \leq\left|q\left(x_{0}\right)\right|+2 \varepsilon C_{k, M}^{-1} \\
& \leq\left|x_{0}(1)\right|^{N-M}\left(\sum_{i=1}^{\infty}\left|x_{0}(i)\right|^{M}\right)+2 \varepsilon C_{k, M}^{-1}<\varepsilon C_{k, M}^{-1}\left(\sum_{i=1}^{\infty} w(i)^{M}+2\right),
\end{aligned}
$$

where $C_{k, M}$ is the constant given in Lemma 4.1. Since $\varepsilon$ was arbitrary we have $\|q\|=0$, which is the desired contradiction.

The following corollary improves [2, Corollary 4.4].

Corollary 4.4. $\operatorname{NAP}_{N}\left(d_{*}(w, 1)\right)$ is dense in $\mathcal{P}_{N}\left(d_{*}(w, 1)\right)$ if and only if $w \notin \ell_{N}$.

Proof. It suffices to prove that $w \notin \ell_{N}$ implies that $\operatorname{NAP}_{N}\left(d_{*}(w, 1)\right)$ is dense in $\mathcal{P}_{N}\left(d_{*}(w, 1)\right)$; the other implication follows from the previous proposition. Note that if $w \notin \ell_{N}$ then $w \notin \ell_{j}$ for all $j \leq N$. As a consequence of [19, Theorem 3.2], we have $\mathcal{P}\left({ }^{j} d_{*}(w, 1)\right)=\mathcal{P}_{\text {wsc }}\left({ }^{j} d_{*}(w, 1)\right)$ for all $j \leq N$ and then $\mathcal{P}_{N}\left(d_{*}(w, 1)\right)=$ $\mathcal{P}_{N, \text { wsc }}\left(d_{*}(w, 1)\right)$. Now, following the arguments in the proof of [19, Theorem 3.2] we obtain the desired result.

Finally, we give some counterexamples in the vector-valued case.

Proposition 4.5. Let $w$ be an admissible sequence and $N \geq 2$.

(i) Suppose $d_{*}(w, 1)$ is a complex Banach space and $W$ is a strictly convex Banach space.

(a) If $w \in \ell_{N}$, then $N A \mathcal{P}_{k}\left(d_{*}(w, 1) ; W\right)$ is not dense in $\mathcal{P}_{k}\left(d_{*}(w, 1) ; W\right)$ for any $k \geq N$.

(b) If $w \in \ell_{r}$ for some $1<r<\infty$, then $N A \mathcal{P}_{k}\left(d_{*}(w, 1)\right.$; $\left.\ell_{r}\right)$ is not dense in $\mathcal{P}_{k}\left(d_{*}(w, 1) ; \ell_{r}\right)$ for any $k \geq 1$.

(ii) If $d_{*}(w, 1)$ is a real Banach space and $w \in \ell_{M} \backslash \ell_{M-1}$ for some $M$ even, then $N A \mathcal{P}_{k}\left(d_{*}(w, 1) ; \ell_{M}\right)$ is not dense in $\mathcal{P}_{k}\left(d_{*}(w, 1) ; \ell_{M}\right)$ for any $k \geq 1$.

Proof. (i) For (a), since $w \in \ell_{N}$, fixing a norm-one element $z_{0} \in W$ we can define $Q \in \mathcal{P}\left({ }^{N} d_{*}(w, 1) ; W\right)$ by

$$
Q(x)=\left(\sum_{i=1}^{\infty} x(i)^{N}\right) z_{0} .
$$

Now the proof follows exactly as in Proposition 4.3. For (b) take $Q(x)=x$, which clearly belongs to $\mathcal{P}_{k}\left(d_{*}(w, 1) ; \ell_{r}\right)$ for every $k \geq 1$, and reason again as in Proposition 4.3 .

(ii) Consider $Q(x)=x$ and suppose that it can be approximated by norm attaining polynomials in $\mathcal{P}_{k}\left(d_{*}(w, 1) ; \ell_{M}\right)$. Since norm-one $M$-homogeneous 
polynomials are uniformly equicontinuous, given $\varepsilon>0$ we can take $P \in$ $N A \mathcal{P}_{k}\left(d_{*}(w, 1) ; \ell_{M}\right)$ so that

$$
\|q \circ Q-q \circ P\|<\varepsilon C_{M k, M}^{-1}
$$

for every norm-one polynomial $q \in \mathcal{P}\left({ }^{M} \ell_{M}\right)$, where $C_{M k, M}$ is the constant given in Lemma 4.1.

Now if $x_{0} \in B_{d_{*}(w, 1)}$ is such that $\left\|P\left(x_{0}\right)\right\|=\|P\|$, we consider the norm-one $M$-homogeneous polynomial $q_{P, x_{0}}: \ell_{M} \rightarrow \mathbb{R}$ given by $q_{P, x_{0}}(x)=\sum_{i=1}^{\infty} x(i)^{M}$. Note that $q_{P, x_{0}} \circ P \in \mathcal{P}_{M k}\left(d_{*}(w, 1)\right)$ is norm attaining and $q_{P, x_{0}} \circ P\left(x_{0}\right)=\|P\|^{M}$. On the other hand, $q_{P, x_{0}} \circ Q(x)=\sum_{i=1}^{\infty} x(i)^{M}$ and by the previous inequality

$$
\left\|\frac{D^{M}\left(q_{P, x_{0}} \circ Q\right)(x)}{M !}-\frac{D^{M}\left(q_{P, x_{0}} \circ P\right)(x)}{M !}\right\|<\varepsilon .
$$

Reasoning as in Proposition 4.3 (here we use the assumption that $M$ is even) we get

$$
\limsup _{n \rightarrow \infty} \frac{D^{M}\left(q_{P, x_{0}} \circ P\right)\left(x_{0}\right)}{M !}\left(e_{n}\right) \leq 0 \text { and } \limsup _{n \rightarrow \infty} \frac{D^{M}\left(q_{P, x_{0}} \circ Q\right)\left(x_{0}\right)}{M !}\left(e_{n}\right)=1 .
$$

Hence

$$
1=\limsup _{n \rightarrow \infty} \frac{D^{M}\left(q_{P, x_{0}} \circ Q\right)\left(x_{0}\right)}{M !}\left(e_{n}\right) \leq \limsup _{n \rightarrow \infty} \frac{D^{M}\left(q_{P, x_{0}} \circ P\right)\left(x_{0}\right)}{M !}\left(e_{n}\right)+\varepsilon \leq \varepsilon,
$$

and since $\varepsilon$ was arbitrary, we obtain the desired contradiction.

In view of Proposition 3.5, it is of interest to find counterexamples to the Bishop-Phelps theorem when the polynomials take values in spaces with property $(\beta)$.

Proposition 4.6. Let $w$ be an admissible sequence and $N \geq 2$.

(i) $N A \mathcal{P}\left({ }^{N} d_{*}(w, 1) ; c_{0}\right)$ is dense in $\mathcal{P}\left({ }^{N} d_{*}(w, 1) ; c_{0}\right)$ if and only if $w \notin \ell_{N}$.

(ii) $N A \mathcal{P}_{N}\left(d_{*}(w, 1) ; c_{0}\right)$ is dense in $\mathcal{P}_{N}\left(d_{*}(w, 1) ; c_{0}\right)$ if and only if $w \notin \ell_{N}$.

Proof. (i) If $w \notin \ell_{N}$ then $N A \mathcal{P}\left({ }^{N} d_{*}(w, 1)\right)$ is dense in $\mathcal{P}\left({ }^{N} d_{*}(w, 1)\right)$, and since $c_{0}$ has property $(\beta)$, by [12, Theorem 2.1] we conclude that $N A \mathcal{P}\left({ }^{N} d_{*}(w, 1) ; c_{0}\right)$ is dense in $\mathcal{P}\left({ }^{N} d_{*}(w, 1) ; c_{0}\right)$.

For the other implication suppose that $w \in \ell_{N}$ and take $Q: d_{*}(w, 1) \rightarrow c_{0}$ defined by

$$
Q(x)=\left(\sum_{i=1}^{\infty} x(i)^{N}, \sum_{i=2}^{\infty} x(i)^{N}, \sum_{i=3}^{\infty} x(i)^{N}, \ldots\right)
$$

in the complex case, and by

$$
Q(x)=x(1)^{N-M}\left(\sum_{i=1}^{\infty}(-1)^{i} x(i)^{M}, \sum_{i=2}^{\infty}(-1)^{i} x(i)^{M}, \sum_{i=3}^{\infty}(-1)^{i} x(i)^{M}, \ldots\right)
$$


in the real case, where $M$ is the smallest natural number such that $w \in \ell_{M}$. Suppose $Q$ can be approximated by norm attaining polynomials in $\mathcal{P}\left({ }^{N} d_{*}(w, 1) ; c_{0}\right)$ and take $P \in N A \mathcal{P}\left({ }^{N} d_{*}(w, 1) ; c_{0}\right)$. Let us see that there exists $m_{0}$ such that $e_{m_{0}}^{*} \circ P$ is norm attaining, where $\left(e_{i}^{*}\right)_{i \in \mathbb{N}}$ is the dual basic sequence of the canonical vectors. Indeed, let $x_{0} \in B_{d_{*}(w, 1)}$ be such that

$$
\left\|P\left(x_{0}\right)\right\|=\sup _{n}\left|e_{n}^{*} \circ P\left(x_{0}\right)\right|=\|P\| .
$$

Since $P\left(x_{0}\right) \in c_{0}$, the supremum in the last equality is actually a maximum, and consequently there exists $m_{0}$ such that $\left|e_{m_{0}}^{*} \circ P\left(x_{0}\right)\right|=\|P\|=\left\|e_{m_{0}}^{*} \circ P\right\|$. Noting that $\left\|e_{m_{0}}^{*} \circ Q-e_{m_{0}}^{*} \circ P\right\| \leq\|Q-P\|$ and reasoning as in [19, Theorem 3.2], we get the desired contradiction.

(ii) The proof is analogous, but reasoning as in Proposition 4.3 instead of [19, Theorem 3.2].

\section{Counterexample in $\mathcal{A}_{u}$}

Let us see that the Bishop-Phelps theorem does not hold for $\mathcal{A}_{u}$ in the vectorvalued case. First we need the following auxiliary lemma. Recall that, as already mentioned in Section 2, in the holomorphic results we consider only complex Banach spaces.

Lemma 4.7. Let $X$ be a Banach sequence space and $W$ be a strictly convex $B a$ nach space. Suppose $x_{0} \in B_{X}$ satisfies the following condition:

$$
\exists n_{0} \in \mathbb{N} \text { and } \delta>0 \text { such that }\left\|x_{0}+\lambda e_{n}\right\| \leq 1, \quad \forall|\lambda| \leq \delta \text { and } n \geq n_{0} .
$$

Then for any $f \in \mathcal{A}_{u}(X ; W)$ and any $n \geq n_{0}$, the function

$$
g_{f}:\{|\lambda|<\delta / 2\} \rightarrow W, \quad \lambda \mapsto f\left(x_{0}+\lambda e_{n}\right),
$$

is holomorphic.

We remark that every element in the unit ball of $c_{0}$ or $d_{*}(w, 1)$ satisfies $(10)$.

Proof. Take a sequence $\left(\alpha_{i}\right)_{i \in \mathbb{N}} \subset \mathbb{R}$ such that $1 / 2<\alpha_{i}<1$ and $\alpha_{i} \nearrow 1$. For $n \geq n_{0}$ we define $g_{i}:\{|\lambda|<\delta / 2\} \rightarrow W$ by

$$
g_{i}(\lambda)=f\left(\alpha_{i} x_{0}+\lambda e_{n}\right) .
$$

Since $\alpha_{i} x_{0}+\lambda e_{n}$ belongs to $\alpha_{i} B_{X}$ for all $|\lambda|<\delta / 2$, the function $g_{i}$ is holomorphic for all $i \geq 1$. Let us show that $g_{i}$ converges uniformly to $g_{f}$. Since $f$ is uniformly continuous, given $\varepsilon>0$ there exists $\delta^{\prime}>0$ such that $\|f(x)-f(y)\|<\varepsilon$ whenever 
$x, y \in B_{X}$ satisfy $\|x-y\|<\delta^{\prime}$. Taking $i$ sufficiently large, we have $1-\alpha_{i}<\delta^{\prime}$ and consequently $\left\|\left(\alpha_{i} x_{0}+\lambda e_{n}\right)-\left(x_{0}+\lambda e_{n}\right)\right\|<\delta^{\prime}$. Then there exists $i_{0}$ such that

$$
\left\|g_{i}(\lambda)-g_{f}(\lambda)\right\|=\left\|f\left(\alpha_{i} x_{0}+\lambda e_{n}\right)-f\left(x_{0}+\lambda e_{n}\right)\right\|<\varepsilon
$$

for all $|\lambda|<\delta / 2$ and all $i \geq i_{0}$. Now, $g_{f}$ is holomorphic since it is the uniform limit of holomorphic functions.

Now, consider the space $Z=c_{0}$ with the norm defined by $\|x\|_{Z}=\|x\|_{\infty}+$ $\left(\sum_{i=1}^{\infty}\left(x(i) / 2^{i}\right)^{2}\right)^{1 / 2}$. Then $\|\cdot\|_{Z}$ and $\|\cdot\|_{\infty}$ are equivalent norms. Moreover, $Z$ and $Z^{\prime \prime}$ are strictly convex. The space $Z$ appears in classical counterexamples to norm attaining results (see for instance [3, 20, 23]).

Proposition 4.8. The set $N A \mathcal{A}_{u}\left(c_{0} ; Z^{\prime \prime}\right)$ is not dense in $\mathcal{A}_{u}\left(c_{0} ; Z^{\prime \prime}\right)$.

Proof. Consider $Q: c_{0} \rightarrow Z^{\prime \prime}$ defined by $Q(x)=x$. It is clear that $Q \in \mathcal{A}_{u}\left(c_{0} ; Z^{\prime \prime}\right)$ and $\left\|Q\left(e_{n}\right)\right\|_{Z^{\prime \prime}} \geq 1$ for all $n \in \mathbb{N}$. Fix $0<\delta<1$, take a norm attaining $f \in \mathcal{A}_{u}\left(c_{0} ; Z^{\prime \prime}\right)$ and let $x_{0} \in B_{c_{0}}$ be such that $\left\|f\left(x_{0}\right)\right\|=\|f\|$. Since $x_{0}$ satisfies condition (10) for the fixed $\delta$ and some $n_{0} \in \mathbb{N}$, by Lemma 4.7 the function

$$
g_{f}:\{|\lambda|<\delta / 2\} \rightarrow Z^{\prime \prime}, \quad g_{f}(\lambda)=f\left(x_{0}+\lambda e_{n}\right),
$$

is holomorphic for fixed $n \geq n_{0}$. Since $g_{f}$ attains its maximum at 0 , it is constant and $D^{j} g_{f}(0)=0$ for all $j \geq 1$. On the other hand, define $g_{Q}(\lambda)=Q\left(x_{0}+\lambda e_{n}\right)$; then $g_{Q}$ is holomorphic and $D^{1} g_{Q}(0)(\lambda)=\lambda Q\left(e_{n}\right)$. Now, by the Cauchy inequalities we obtain

$$
1 \leq\left\|D^{1} g_{Q}(0)-D^{1} g_{f}(0)\right\| \leq \frac{1}{\delta / 2} \sup _{|\lambda|<\delta / 2}\left\|g_{Q}(\lambda)-g_{f}(\lambda)\right\| \leq \frac{2}{\delta}\|Q-f\| .
$$

Hence, $Q$ cannot be approximated by norm attaining functions in $\mathcal{A}_{u}\left(c_{0} ; Z^{\prime \prime}\right)$.

It is worth noting that the argument in the previous proof does not work if we consider functions defined on $d_{*}(w, 1)$ (instead of $c_{0}$ ) with values in a strictly convex Banach space. The reason is that, although any $x_{0} \in B_{d_{*}(w, 1)}$ satisfies condition (10), we cannot fix $\delta$ independently of $f$. In fact, in this case $\delta$ depends on $x_{0}$ and can be arbitrarily small.

\section{Counterexamples to strong versions of the Bishop-Phelps theorem}

We have already mentioned that we do not know whether the Bishop-Phelps theorem holds for $\mathcal{A}_{u}$ in the scalar-valued case. We now show that the strong versions of this theorem introduced in [2] which we studied in Section 3 actually fail, while the corresponding strong versions of the Lindenstrauss theorem hold. For this purpose, we state the next lemma which is analogous to Lemma 4.2. 
Lemma 4.9. Let $X$ be a Banach sequence space and $W$ be a strictly convex Banach space. Let $0<s<1$ and $f \in \mathcal{A}_{u}(X ; W)$.

(i) Fix $0<s_{0}<1$ and consider $x_{0} \in s B_{X}$ for some $0<s<s_{0}$. Then

$$
\left\|\frac{D^{j} f\left(x_{0}\right)}{j !}\right\| \leq \frac{1}{\left(s_{0}-s\right)^{j}}\|f\|_{s_{0}}
$$

for all $j \geq 1$.

(ii) Suppose that $f$ attains its $\|\cdot\|_{s}$-norm at an element $x_{0} \in s B_{X}$ satisfying the following condition:

(11) $\exists n_{0} \in \mathbb{N}$ and $\delta>0$ such that $\left\|x_{0}+\lambda e_{n}\right\| \leq s, \quad \forall|\lambda| \leq \delta$ and $n \geq n_{0}$.

Then $D^{j} f\left(x_{0}\right)\left(e_{n}\right)=0$ for all $j \geq 1$ and $n \geq n_{0}$.

Proof. (i) Fix $r=s_{0}-\left\|x_{0}\right\|$ and $y \in B_{X}^{\mathrm{o}}$, and consider the one-variable holomorphic function

$$
g_{f}:\{|\lambda|<1\} \rightarrow W, \quad \lambda \mapsto f\left(x_{0}+\lambda r y\right) .
$$

By the Cauchy inequalities we have

$$
\left\|\frac{D^{j} g_{f}(0)}{j !}\right\| \leq \sup _{|\lambda|<1}\left\|g_{f}(\lambda)\right\| \leq\|f\|_{s_{0}}
$$

for all $j \geq 1$. Now, noting that $D^{j} g_{f}(0)=r^{j} D^{j} f\left(x_{0}\right)(y)$ we deduce

$$
r^{j}\left\|\frac{D^{j} f\left(x_{0}\right)(y)}{j !}\right\| \leq\|f\|_{s_{0}}
$$

and since $y \in B_{X}^{\mathrm{o}}$ was arbitrary, the desired statement follows.

(ii) Since $\left\|x_{0}\right\| \leq s<1$ and $f$ is holomorphic in $B_{X}^{\circ}$, we can consider the series expansion of $f$ at $x_{0}$,

$$
f(x)=\sum_{j=0}^{\infty} \frac{D^{j} f\left(x_{0}\right)}{j !}\left(x-x_{0}\right) .
$$

Then the statement follows by evaluating at $x=x_{0}+\lambda e_{n}$ with $n \geq n_{0}$ and proceeding as in Lemma 4.2

We remark that, as expected, every element in the unit ball of $c_{0}$ or $d_{*}(w, 1)$ satisfies condition (11). The following result is the improvement of [2, Corollary 4.5] mentioned above. Both Lemma 4.9 and Proposition 4.10 hold with $H^{\infty}\left(B_{d_{*}(w, 1)}^{\mathrm{o}} ; W\right)$ in place of $\mathcal{A}_{u}\left(d_{*}(w, 1) ; W\right)$. 
Proposition 4.10. Let $W$ be a strictly convex space and take an admissible sequence $w \in \ell_{N}$ for some $N \geq 2$. Given $0<s<s_{0} \leq 1$, there exists an $N$ homogeneous polynomial that cannot be approximated in the $\|\cdot\|_{s_{0}}$-norm (in particular, in the $\|\cdot\|-n o r m)$ by elements of $\mathcal{A}_{u}\left(d_{*}(w, 1) ; W\right)$ that attain the $\|\cdot\|_{s}$-norm.

Proof. Fix a norm-one element $z_{0} \in W$ and define $Q: d_{*}(w, 1) \rightarrow W$ by

$$
Q(x)=\left(\sum_{i=1}^{\infty} x(i)^{N}\right) z_{0} .
$$

Then $Q$ is in $\mathcal{P}\left({ }^{N} d_{*}(w, 1) ; W\right)$ and its restriction to the ball belongs to $\mathcal{A}_{u}\left(d_{*}(w, 1) ; W\right)$. Take $f \in \mathcal{A}_{u}\left(d_{*}(w, 1) ; W\right)$ that attains its $\|\cdot\|_{s}$-norm at some $x_{0} \in s B_{d_{*}(w, 1)}$. By Lemma 4.9(ii), there exists $n_{0} \in \mathbb{N}$ such that $D^{j} f\left(x_{0}\right)\left(e_{n}\right)=0$ for all $j \geq 1$ and $n \geq n_{0}$. On the other hand, $D^{N} Q\left(x_{0}\right) / N !=Q$ and hence $\left\|\left(D^{N} Q\left(x_{0}\right) / N !\right)\left(e_{n}\right)\right\|=1$ for all $n \in \mathbb{N}$. For $n \geq n_{0}$, by Lemma 4.9(i) we have

$$
1=\left\|\frac{D^{N} Q\left(x_{0}\right)}{N !}\left(e_{n}\right)-\frac{D^{N} f\left(x_{0}\right)}{N !}\left(e_{n}\right)\right\| \leq \frac{1}{\left(s_{0}-s\right)^{N}}\|Q-f\|_{s_{0}} .
$$

Therefore, $Q$ cannot be approximated by a function $f \in \mathcal{A}_{u}\left(d_{*}(w, 1) ; W\right)$ that attains its $\|\cdot\|_{s}$-norm. This gives the desired statement.

Finally, we have the following equivalence in the spirit of Proposition 4.3. See also [4, Proposition 2.6] where, with the same tools, a similar equivalence is given.

Corollary 4.11. Let $0<s<1$. The set of functions in $\mathcal{A}_{u}\left(d_{*}(w, 1)\right)$ attaining their $\|\cdot\|_{s}$-norm is $\|\cdot\|$-dense in $\mathcal{A}_{u}\left(d_{*}(w, 1)\right)$ if and only if $w \notin \ell_{N}$ for all $N \in \mathbb{N}$.

Proof. First note that we can proceed as in Corollary 4.4 to show that for $0<$ $s<1$, the set of $\|\cdot\|_{s}$-norm attaining polynomials in $\mathcal{P}_{N}\left(d_{*}(w, 1)\right)$ is $\|\cdot\|$-dense in $\mathcal{P}_{N}\left(d_{*}(w, 1)\right)$ if and only if $w \notin \ell_{N}$. This implies, if $w \notin \ell_{N}$ for all $N \in \mathbb{N}$, that the set of $\|\cdot\|_{s}$-norm attaining polynomials is dense in the space $\mathcal{P}\left(d_{*}(w, 1)\right)$ of polynomials (of any degree). Then, given $g \in \mathcal{A}_{u}\left(d_{*}(w, 1)\right)$ and $\varepsilon>0$, we can take a polynomial $q$ such that $\|g-q\|<\varepsilon / 2$, and then a $\|\cdot\|_{s}$-norm attaining polynomial $p$ such that $\|q-p\|<\varepsilon / 2$. This proves one implication, while the other follows from Proposition 4.10.

As a consequence, taking $\mathcal{A}_{u}\left(d_{*}(w, 1)\right)$ with $w \in \ell_{r}$ for some $1<r<\infty$ we obtain, in the scalar-valued case, the desired examples of spaces for which the strong version of the Bishop-Phelps theorem fails, but the corresponding strong version of the Lindenstrauss theorem holds. For arbitrary admissible sequences (which need not belong to any $\ell_{r}$ ), we can do the following in the vector-valued case. We take again a renorming $Z$ of $c_{0}$ such that its bidual $Z^{\prime \prime}$ is strictly convex, 
and consider $Q(x)=x$ which is well defined from $d_{*}(w, 1)$ to $Z^{\prime \prime}$ whether $w$ belongs to some $\ell_{r}$ or not. Moreover, $Q$ is well defined from $c_{0}$ to $Z^{\prime \prime}$ and the strong version of the Bishop-Phelps theorem fails also in this case since the Bishop-Phelps theorem fails according to Proposition 4.8.

As in the polynomial case, we also have the previous equivalence when we consider holomorphic functions with values in $c_{0}$. Indeed, with slight modifications in the proof of [12, Theorem 2.1], we find that if the strong version of Bishop-Phelps holds for $\mathcal{A}_{u}\left(d_{*}(w, 1)\right)$ and $Y$ has property $(\beta)$ then it holds for $\mathcal{A}_{u}\left(d_{*}(w, 1) ; Y\right)$. For the other implication, the same polynomial $Q$ of Proposition 4.6 works as a counterexample and the proof follows the same lines, making use of Lemma 4.9 to prove that

$$
1=\left|\frac{D^{N}\left(e_{m_{0}}^{*} \circ Q\right)\left(x_{0}\right)}{N !}\left(e_{n}\right)-\frac{D^{N}\left(e_{m_{0}}^{*} \circ f\right)\left(x_{0}\right)}{N !}\left(e_{n}\right)\right| \leq \frac{1}{(1-s)^{N}}\|Q-f\|
$$

for any $\|\cdot\|_{s}$-norm attaining $f \in \mathcal{A}_{u}\left(d_{*}(w, 1) ; c_{0}\right)$ and $n$ large enough. This gives the desired statement.

\section{Acknowledgements}

This project was partially supported by CONICET PIP 0624, PICT 2011-1456 and UBACyT 20020130100474BA. The second author is supported by a doctoral fellowship from CONICET.

\section{References}

[1] M. D. Acosta, F. J. Aguirre, and R. Payá, There is no bilinear Bishop-Phelps theorem, Israel J. Math. 93 (1996), 221-227. Zbl 0852.46010 MR 1380644

[2] M. D. Acosta, J. Alaminos, D. García, and M. Maestre, On holomorphic functions attaining their norms, J. Math. Anal. Appl. 297 (2004), 625-644. Zbl 1086.46034 MR 2088685

[3] M. D. Acosta, R. M. Aron, D. García, and M. Maestre, The Bishop-Phelps-Bollobás theorem for operators, J. Funct. Anal. 254 (2008), 2780-2799. Zbl 1152.46006 MR 2414220

[4] M. D. Acosta, R. M. Aron, and L. A. Moraes, Boundaries for spaces of holomorphic functions on $M$-ideals in their biduals, Indiana Univ. Math. J. 58 (2009), 2575-2595. Zbl 1193.46031 MR 2603760

[5] M. D. Acosta, D. García, and M. Maestre, A multilinear Lindenstrauss theorem, J. Funct. Anal. 235 (2006), 122-136. Zbl 1101.46029 MR 2216442

[6] R. Arens, The adjoint of a bilinear operation, Proc. Amer. Math. Soc. 2 (1951), 839-848. Zbl 0044.32601 MR 0045941

[7] R. M. Aron and P. D. Berner, A Hahn-Banach extension theorem for analytic mappings, Bull. Soc. Math. France 106 (1978), 3-24. Zbl 0378.46043 MR 0508947

[8] R. M. Aron, D. García, and M. Maestre, On norm attaining polynomials, Publ. RIMS Kyoto Univ. 39 (2003), 165-172. Zbl 1035.46005 MR 1935463

[9] E. Bishop and R. R. Phelps, A proof that every Banach space is subreflexive, Bull. Amer. Math. Soc. 67 (1961), 97-98. Zbl 0098.07905 MR 0123174 
[10] The support functionals of a convex set, in Convexity, Proc. Sympos. Pure Math. 7, Amer. Math. Soc., Providence, RI, 1963, 27-35. Zbl 0149.08601 MR 0154092

[11] D. Carando, S. Lassalle, and M. Mazzitelli, On the polynomial Lindenstrauss theorem, J. Funct. Anal. 263 (2012), 1809-1824. Zbl 1258.46017 MR 2956926

[12] Y. S. Choi and S. G. Kim, Norm or numerical radius attaining multilinear mappings and polynomials, J. London Math. Soc. (2) 54 (1996), 135-147. Zbl 0858.47005 MR 1395073

[13] Y. S. Choi, H. J. Lee, and H. G. Song, Denseness of norm-attaining mappings on Banach spaces, Publ. RIMS Kyoto Univ. 46 (2010), 171-182. Zbl 1194.46010 MR 2662617

[14] A. M. Davie and T. W. Gamelin. A theorem on polynomial-star approximation, Proc. Amer. Math. Soc. 106 (1989), 351-356. Zbl 0683.46037 MR 0947313

[15] A. Defant and K. Floret, Tensor norms and operator ideals, North-Holland Math. Stud. 176. North-Holland, Amsterdam, 1993. Zbl 0774.46018 MR 1209438

[16] W. T. Gowers, Symmetric block bases of sequences with large average growth, Israel J. Math. 69 (1990), 129-151. Zbl 0721.46010 MR 1045369

[17] L. A. Harris, Bounds on the derivatives of holomorphic functions of vectors, in Analyse fonctionnelle et applications (Rio de Janeiro, 1972), Actualités Sci. Indust. 1367, Hermann, Paris, 1975, 145-163. Zbl 0315.46040 MR 0477773

[18] _ Markov's inequality for polynomials on normed linear spaces, Math. Balkanica (N.S.) 16 (2002), 315-326. Zbl 1068.41025 MR 1934000

[19] M. Jiménez Sevilla and R. Payá, Norm attaining multilinear forms and polynomials on preduals of Lorentz sequence spaces, Studia Math. 127 (1998), 99-112. Zbl 0909.46015 MR 1488146

[20] J. Lindenstrauss, On operators which attain their norm, Israel J. Math. 1 (1963), 139-148. Zbl 0127.06704 MR 0160094

[21] J. Lindenstrauss and L. Tzafriri, Classical Banach spaces I. Sequence spaces, Ergeb. Math. Grenzgeb. 92, Springer, Berlin, 1977. Zbl 0362.46013 MR 0500056

[22] J. Mujica, Linearization of bounded holomorphic mappings on Banach spaces, Trans. Amer. Math. Soc. 324 (1991), 867-887. Zbl 0747.46038 MR 1000146

[23] R. Payá, A counterexample on numerical radius attaining operators, Israel J. Math. 1 (1992), 83-101. Zbl 0784.47005 MR 1195254 